\title{
A SUBCLASS OF HARMONIC UNIVALENT FUNCTION INVOLVING ERROR FUNCTION
}

\author{
I.T. AWOLERE ${ }^{1, *}$, UKEJE EMELIKE $^{2}$ \\ ${ }^{1}$ Department of Mathematics, Emmanuel Alayande College of Education, P. M. B. 1010, Oyo, Oyo State, Nigeria \\ ${ }^{2}$ Department of Mathematics, Micheal Okpara University of Agiculture, Umudike, Abia State, Nigeria \\ Corresponding author: awolereibrahim01@gmail.com \\ Received Sept. 1, 2018
}

\begin{abstract}
AвSTRACT. In this investigation we introduce and study a subclass of harmonic univalent functions associated with error function and salagean operator using convolutional approach. Coefficient estimate, distortion bounds, growth theorem, extreme points and convex combination were established for a new class $\bar{T}_{H, n}(\lambda)$. Consequently, our results are new approaches to those corresponding to previously known results.
\end{abstract}

2010 Mathematics Subject Classification. Primary 30A10, Secondary 26 D07.

Key words and phrases. analytic functions; coefficient inequalities; univalent functions; harmonic function; error functions; convex functions.

\section{INTRODUCTION}

The theory of special functions are significantly inportant to scientist and engineers with mathematically calculations [Oladipo, 10].The special function does not have specific definition but its applications extends to physics, computer e.t.c. There are various special functions but we shall concern ourselves with error function which has application in probability, statistics,materials science and partial differential equations. Abramowitz el al[1] defined error function as

$$
\operatorname{erf}(z)=\frac{2}{\sqrt{\pi}} \int_{0}^{z} e^{-t^{2}} d t=\frac{2}{\sqrt{\pi}} \sum_{k=2}^{\infty} \frac{(-1)^{k-1} z^{2 k+1}}{(2 k+1) k !}
$$

For detailed about properties and inequalities error function and properties of complementary error function see $[3,5,7]$.

We shall denote by $A$ the class of the form

$$
f(z)=z+\sum_{k=2}^{\infty} a_{k} z^{k}
$$

which are analytic in the open unit disk $U=\{z:|z|, z \in U\}$ nt functions with normalization $f(0)=f^{\prime}(0)-1=0$. Also, let $S$ be the subclass of $A$ consisting of univalent functions in $U$.

A function $f \in A$ is said to be in the class $S^{*}$ and class $C$ of starlike and convex in $A$, if it satisfies the following inequalities respectifully

$$
\operatorname{Re}\left(\frac{z f^{\prime}(z)}{f(z)}\right)>0, \quad z \in U
$$

DOI: 10.28924/APJM/6-9 


$$
\operatorname{Re}\left(1+\frac{z f^{\prime \prime}(z)}{f^{\prime}(z)}\right)>0, \quad z \in U
$$

Let $E$ be the class of modified error function which was intrduced and studied by Ramchandran et al [11]as

$$
E_{r} f(z)=\frac{\sqrt{\pi z}}{2} \operatorname{erf}(\sqrt{z})=z+\sum_{k=2}^{\infty} \frac{(-1)^{k-1}}{(2 k-1)(k-1) !} z^{k}
$$

where $E_{r} f$ is anormalized analytic function which is obtained from (1) A continous complex-valued function $f=u+i v$ is said to be harmonic in a simply-connected domain $D$ if both $u$ and $v$ are real harmonic in $D$. Various classes of harmonic functions have been extensively investigated in the literature of the subject for example, see $[2,6,8,13]$ and other references there in. The function $f=h+\bar{g}$ is said to be harmonic univalent in $D$ if the mapping $z \rightarrow f(z)$ is orietation preserving, harmonic and one-to-one in $D$. We call $h$ the analytic part and $g$ the co-analytic off. For detailed see Clunie and Sheil-Small [4].

Denote by $H$ the class of the class of the functions that are harmonic univalent and orientation preserving in the open unit disk $U=\{z:|z|<1\}$ for which $f(0)=f_{z}(0)-1=0$. Then for $f=h+\bar{g} \in H$, we may express the analytic functions $f$ and $g$ as

A function $f=h+\bar{g}$ with $h$ and $g$ be given by (1) is said to be harmonic starlike of order $\beta, 0 \leq \beta<1$ for $|z|=r<1$, if

$$
\frac{\partial}{\partial \theta}\left(\arg f\left(r e^{i \theta}\right)\right)=R\left\{\frac{z h^{\prime}(z)-z \overline{z g^{\prime}(z)}}{h(z)+\bar{g}(z)}\right\} \geq \beta
$$

The class of all harmonic starlike of order $\beta$ is denoted by $S_{H}^{*}(\beta)$ and vigoriousily study by jahangiri [9].

The convolution of two power series

$$
D^{n}(f)(z)=z-\sum_{k=2}^{\infty} k^{n} a_{k} z^{k}
$$

and

$$
E_{r} f(z)=z-\sum_{k=2}^{\infty} \frac{(-1)^{k-1}}{(2 k-1)(k-1) !} z^{k}
$$

is defined by

$$
\left(D^{n} * E_{r}\right)(z)=z-\sum_{k=2}^{\infty} \frac{k^{n}(-1)^{k-1}}{(2 k-1)(k-1) !} a_{k} z^{k}
$$

where $D^{n}$ isderivative operator defined by Salagean [12]

Definition 1:Let $D^{n} E_{r} f=D^{n} E_{r} h+\overline{D^{n} E_{r} g}$ with $h$ and $g$ be given by (1) where

$$
\begin{gathered}
D^{n} E_{r} h(z)=z-\sum_{k=2}^{\infty} \frac{k^{n}(-1)^{k-1}}{(2 k-1)(k-1) !} a_{k} z^{k}, \\
D^{n} E_{r} g(z)=\sum_{k=1}^{\infty} \frac{k^{n}(-1)^{k-1}}{(2 k-1)(k-1) !} \overline{b_{k}} z^{k}, \quad\left|b_{1}\right|<1 .
\end{gathered}
$$

Then $f \in \bar{T}_{H, n}(\lambda)$ if and only if , for $\beta, 0 \leq \beta<1$ and $[-1,1]$

$$
\operatorname{Re}\left(\frac{z D^{n} \operatorname{Erf}^{\prime}(z)-\overline{z D^{n} \operatorname{Erg}^{\prime}(z)}}{D^{n} \operatorname{Erf}(z)+\overline{D^{n} \operatorname{Erg}(z)}}-\lambda\right) \leq\left|\frac{z D^{n} \operatorname{Erf} f^{\prime}(z)-\overline{z D^{n} \operatorname{Erg}^{\prime}(z)}}{D^{n} \operatorname{Erf}(z)+\overline{D^{n} \operatorname{Erg}(z)}}-1\right|
$$


Theorem 1:Let $f \in \bar{T} S_{H, n}(\lambda)$ then

$$
\sum_{k=2}^{\infty} \frac{k^{n}(-1)^{k-1}}{(2 k-1)(k-1) !}[2 k-\lambda-1]\left|a_{k}\right|+\sum_{k=1}^{\infty} \frac{k^{n}(-1)^{k-1}}{(2 k-1)(k-1) !}[2 k+\lambda+1]\left|b_{\bar{k}}\right| \leq 1-\lambda
$$

Proof:Let $f \in \bar{T}_{H, n}(\lambda)$ with $\lambda \in[-1,1]$. We have

$$
\operatorname{Re}\left(\frac{z D^{n} \operatorname{Erf}^{\prime}(z)-\overline{z D^{n} \operatorname{Erg}^{\prime}(z)}}{D^{n} \operatorname{Erf}(z)+\overline{D^{n} \operatorname{Erg}(z)}}-\lambda\right) \leq\left|\frac{z D^{n} \operatorname{Erf} f^{\prime}(z)-\overline{z D^{n} \operatorname{Erg}(z)}}{D^{n} \operatorname{Erf}(z)+\overline{D^{n} \operatorname{Erg}(z)}}-1\right|
$$

If we take $z \in[0,1]$, we have

$$
\begin{gathered}
\frac{1-\sum_{k=2}^{\infty} \frac{k^{n+1}(-1)^{k-1}}{(2 k-1)(k-1) !} a_{k} z^{k-1}-\sum_{k=1}^{\infty} \frac{k^{n+1}(-1)^{k-1}}{(2 k-1)(k-1) !} \overline{b_{k}} z^{k-1}}{1-\sum_{k=2}^{\infty} \frac{k^{n}(-1)^{k-1}}{(2 k-1)(k-1) !} a_{k} z^{k-1}+\sum_{k=1}^{\infty} \frac{k^{n}(-1)^{k-1}}{(2 k-1)(k-1) !} \overline{b_{k}} z^{k-1}}-\lambda \\
\geq 1-\frac{1-\sum_{k=2}^{\infty} \frac{k^{n+1}(-1)^{k-1}}{(2 k-1)(k-1) !} a_{k} z^{k-1}-\sum_{k=1}^{\infty} \frac{k^{n+1}(-1)^{k-1}}{(2 k-1)(k-1) !} \overline{b_{k}} z^{k-1}}{1-\sum_{k=2}^{\infty} \frac{k^{n}(-1)^{k-1}}{(2 k-1)(k-1) !} a_{k} z^{k-1}+\sum_{k=1}^{\infty} \frac{k^{n}(-1)^{k-1}}{(2 k-1)(k-1) !} \overline{b_{k}} z^{k-1}}
\end{gathered}
$$

This yields

$$
\sum_{k=2}^{\infty} \frac{k^{n}(-1)^{k-1}}{(2 k-1)(k-1) !}[2 k-\lambda-1]\left|a_{k}\right|+\sum_{k=1}^{\infty} \frac{k^{n}(-1)^{k-1}}{(2 k-1)(k-1) !}[2 k+\lambda+1]\left|b_{\bar{k}}\right| \leq 1-\lambda
$$

Letting $z \rightarrow 1^{-1}$ along the real axis we have

$$
\sum_{k=2}^{\infty} \frac{k^{n}(-1)^{k-1}}{(2 k-1)(k-1) !}[2 k-\lambda-1]\left|a_{k}\right|+\sum_{k=1}^{\infty} \frac{k^{n}(-1)^{k-1}}{(2 k-1)(k-1) !}[2 k+\lambda+1]\left|b_{\bar{k}}\right|<1-\lambda
$$

Conversely, let us take $f(z) \in T$ for which the relation () hold. It suffices to show that

$$
\left|\frac{z D^{n} \operatorname{Erf}^{\prime}(z)-\overline{z D^{n} \operatorname{Erg}^{\prime}(z)}}{D^{n} \operatorname{Erf}(z)+\overline{D^{n} \operatorname{Erg}(z)}}-1\right|-\operatorname{Re}\left\{\frac{z D^{n} \operatorname{Erf}^{\prime}(z)-\overline{z D^{n} \operatorname{Erg}^{\prime}(z)}}{D^{n} \operatorname{Erf}(z)+\overline{D^{n} \operatorname{Erg}(z)}}-1\right\} \leq 1-\lambda
$$

We have

$$
\begin{aligned}
& \left|\frac{z D^{n} \operatorname{Erf}^{\prime}(z)-\overline{z D^{n} \operatorname{Erg}^{\prime}(z)}}{D^{n} \operatorname{Erf}(z)+\overline{D^{n} \operatorname{Erg}(z)}}-1\right|-\operatorname{Re}\left\{\frac{z D^{n} \operatorname{Erf} f^{\prime}(z)-\overline{z D^{n} \operatorname{Erg}^{\prime}(z)}}{D^{n} \operatorname{Erf}(z)+\overline{D^{n} \operatorname{Erg}(z)}}-1\right\} \leq \\
& \quad 2\left|\frac{z D^{n} \operatorname{Erf}^{\prime}(z)-\overline{z D^{n} \operatorname{Erg}^{\prime}(z)}}{D^{n} \operatorname{Erf}(z)+\overline{D^{n} \operatorname{Erg}(z)}}-1\right| \\
& \quad=2\left|\frac{z D^{n} \operatorname{Erf}^{\prime}(z)-\overline{z D^{n} \operatorname{Erg}(z)}-D^{n} \operatorname{Erf}(z)-\overline{D^{n} \operatorname{Erg}(z)}}{D^{n} \operatorname{Erf}(z)+\overline{D^{n} \operatorname{Erg}(z)}}\right| \\
& \leq \frac{1-\sum_{k=2}^{\infty} \frac{2 k^{n}(-1)^{k-1}}{(2 k-1)(k-1) !}[k-1] a_{k}+\sum_{k=1}^{\infty} \frac{2 k^{n}(-1)^{k-1}}{(2 k-1)(k-1) !}[k+1] a_{k}}{1-\sum_{k=2}^{\infty} \frac{k^{n+1}(-1)^{k-1}}{(2 k-1)(k-1) !} a_{k} z^{k-1}-\sum_{k=1}^{\infty} \frac{k^{n+1}(-1)^{k-1}}{(2 k-1)(k-1) !} \overline{b_{k}} z^{k-1}}
\end{aligned}
$$

The last expression is bounded above by $1-\lambda$ if

$$
\sum_{k=2}^{\infty} \frac{k^{n}(-1)^{k-1}}{(2 k-1)(k-1) !}[2 k-\lambda-1]\left|a_{k}\right|+\sum_{k=1}^{\infty} \frac{k^{n}(-1)^{k-1}}{(2 k-1)(k-1) !}[2 k+\lambda+1]\left|b_{\bar{k}}\right| \leq 1-\lambda
$$


If we choose $\lambda=0$ in the theorem above,yields Corrolary 1 below:

Corrolary 1:Let $f \in \bar{T} S_{H, n}(0)$ then

$$
\sum_{k=2}^{\infty} \frac{k^{n}(-1)^{k-1}}{(2 k-1)(k-1) !}[2 k-1]\left|a_{k}\right|+\sum_{k=1}^{\infty} \frac{k^{n}(-1)^{k-1}}{(2 k-1)(k-1) !}[2 k+1]\left|b_{\bar{k}}\right| \leq 1
$$

Theorem 2:Let $f \in \bar{T}_{H, n}(\lambda)$ with $\lambda \in[-1,1]$. We have

$$
\begin{gathered}
|f(z)| \leq\left(1+\left|b_{1}\right|\right) r-\frac{3}{2^{n}}\left[\frac{1-\lambda}{3-\lambda}-\frac{3+\lambda}{3-\lambda}\right] r^{2} \\
|f(z)| \geq\left(1+\left|b_{1}\right|\right) r+\frac{3}{2^{n}}\left[\frac{1-\lambda}{3-\lambda}-\frac{3+\lambda}{3-\lambda}\right] r^{2} \quad \text { where }\left|b_{1}\right| \leq \frac{1-\lambda}{3+\lambda}
\end{gathered}
$$

The result is sharp

Proof:We shall prove the first inequality. Let $f \in \bar{T}_{H, n}(\lambda)$. Then we have

$$
\begin{gathered}
|f(z)| \geq\left(1+\left|b_{1}\right|\right) r+\sum_{k=2}^{\infty}\left(\left|a_{k}\right|+\left|b_{k}\right|\right) r^{k} \leq\left(1+\left|b_{1}\right|\right) r+r^{2} \sum_{k=2}^{\infty}\left(\left|a_{k}\right|+\left|b_{k}\right|\right) \\
=\left(1+\left|b_{1}\right|\right) r-\frac{3(1-\lambda)}{2^{n}(3-\lambda)} \sum_{k=2}^{\infty} \frac{2^{n}(3-\lambda)}{3(1-\lambda)}\left(\left|a_{k}\right|+\left|b_{k}\right|\right) r^{2}
\end{gathered}
$$

and so

$$
\begin{aligned}
|f(z)| \leq\left(1+\left|b_{1}\right|\right) r & -\frac{3(1-\lambda)}{2^{n}(3-\lambda)} \sum_{k=2}^{\infty} A\left[\frac{2 k-\lambda-1}{1-\lambda}\left|a_{k}\right|+\frac{2 k+\lambda+1}{1-\lambda}\left|b_{k}\right|\right] r^{2} \\
\leq & \left(1+\left|b_{1}\right|\right) r-\frac{3(1-\lambda)}{2^{n}(3-\lambda)}\left[1-\frac{3+\lambda}{1-\lambda}\left|b_{1}\right|\right] r^{2} \\
= & \leq\left(1+\left|b_{1}\right|\right) r-\frac{3}{2^{n}}\left[\frac{1-\lambda}{3-\lambda}-\frac{3+\lambda}{3-\lambda}\right] r^{2} \\
& =\leq\left(1+\left|b_{1}\right|\right) r-\frac{3}{2^{n}}\left[\frac{1-\lambda}{3-\lambda}-\frac{3+\lambda}{3-\lambda}\right] r^{2}
\end{aligned}
$$

where $A=\frac{k^{n}(-1)^{k-1}}{(2 k-1)(k-1) !}$

On the other hand

$$
\begin{gathered}
|f(z)| \geq\left(1+\left|b_{1}\right|\right) r-\sum_{k=2}^{\infty}\left(\left|a_{k}\right|+\left|b_{k}\right|\right) r^{k} \leq\left(1+\left|b_{1}\right|\right) r+r^{2} \sum_{k=2}^{\infty}\left(\left|a_{k}\right|+\left|b_{k}\right|\right) \\
=\left(1+\left|b_{1}\right|\right) r+\frac{3(1-\lambda)}{2^{n}(3-\lambda)} \sum_{k=2}^{\infty} \frac{2^{n}(3-\lambda)}{3(1-\lambda)}\left(\left|a_{k}\right|+\left|b_{k}\right|\right) r^{2}
\end{gathered}
$$

and so

$$
\begin{gathered}
|f(z)| \geq\left(1+\left|b_{1}\right|\right) r+\frac{3(1-\lambda)}{2^{n}(3-\lambda)} \sum_{k=2}^{\infty} A\left[\frac{2 k-\lambda-1}{1-\lambda}\left|a_{k}\right|+\frac{2 k+\lambda+1}{1-\lambda}\left|b_{k}\right|\right] r^{2} \\
\geq\left(1+\left|b_{1}\right|\right) r+\frac{3(1-\lambda)}{2^{n}(3-\lambda)}\left[1-\frac{3+\lambda}{1-\lambda}\left|b_{1}\right|\right] r^{2} \\
=\left(1+\left|b_{1}\right|\right) r+\frac{3}{2^{n}}\left[\frac{1-\lambda}{3-\lambda}-\frac{3+\lambda}{3-\lambda}\right] r^{2}
\end{gathered}
$$




$$
=\left(1+\left|b_{1}\right|\right) r+\frac{3}{2^{n}}\left[\frac{1-\lambda}{3-\lambda}-\frac{3+\lambda}{3-\lambda}\right] r^{2}
$$

which complete the proof.

Theorem 3:Let $f \in \bar{T}_{H, n}(\lambda)$ with $\lambda \in[-1,1]$. We have

$$
\begin{gathered}
\left|f^{\prime}(z)\right| \leq 1+\left|b_{1}\right|-\frac{6}{2^{n}}\left[\frac{1-\lambda}{3-\lambda}-\frac{3+\lambda}{3-\lambda}\right] r \\
\left|f^{\prime}(z)\right| \geq\left(1+\left|b_{1}\right|\right) r+\frac{6}{2^{n}}\left[\frac{1-\lambda}{3-\lambda}-\frac{3+\lambda}{3-\lambda}\right] r \quad \text { where }\left|b_{1}\right| \leq \frac{1-\lambda}{3+\lambda}
\end{gathered}
$$

The result is sharp

Proof:We shall prove the first inequality. Let $f \in \bar{T}_{H, n}(\lambda)$. Then we have

$$
\begin{gathered}
\left|f^{\prime}(z)\right| \geq 1+\left|b_{1}\right|+\sum_{k=2}^{\infty} k\left(\left|a_{k}\right|+\left|b_{k}\right|\right) r^{k} \geq 1+\left|b_{1}\right|+r \sum_{k=2}^{\infty} k\left(\left|a_{k}\right|+\left|b_{k}\right|\right) \\
=1+\left|b_{1}\right|-\frac{6(1-\lambda)}{2^{n}(3-\lambda)} \sum_{k=2}^{\infty} \frac{2^{n}(3-\lambda)}{6(1-\lambda)}\left(\left|a_{k}\right|+\left|b_{k}\right|\right) r
\end{gathered}
$$

and so

$$
\begin{gathered}
|f(z)| \geq 1+\left|b_{1}\right|-\frac{6(1-\lambda)}{2^{n}(3-\lambda)} \sum_{k=2}^{\infty} A\left[\frac{2 k-\lambda-1}{1-\lambda}\left|a_{k}\right|+\frac{2 k+\lambda+1}{1-\lambda}\left|b_{k}\right|\right] r \\
\leq 1+\left|b_{1}\right|-\frac{3(1-\lambda)}{2^{n}(3-\lambda)}\left[1-\frac{3+\lambda}{1-\lambda}\left|b_{1}\right|\right] r \\
=1+\left|b_{1}\right|-\frac{6}{2^{n}}\left[\frac{1-\lambda}{3-\lambda}-\frac{3+\lambda}{3-\lambda}\right] r \\
=\left(1+\left|b_{1}\right|-\frac{6}{2^{n}}\left[\frac{1-\lambda}{3-\lambda}-\frac{3+\lambda}{3-\lambda}\right] r\right.
\end{gathered}
$$

where $A=\frac{k^{n}(-1)^{k-1}}{(2 k-1)(k-1) !}$

On the other hand

$$
\begin{gathered}
\left|f^{\prime}(z)\right| \geq 1+\left|b_{1}\right|+\sum_{k=2}^{\infty} k\left(\left|a_{k}\right|+\left|b_{k}\right|\right) r^{k} \geq\left(1+\left|b_{1}\right|\right)+r \sum_{k=2}^{\infty} k\left(\left|a_{k}\right|+\left|b_{k}\right|\right) \\
=1+\left|b_{1}\right|-\frac{6(1-\lambda)}{2^{n}(3-\lambda)} \sum_{k=2}^{\infty} \frac{2^{n}(3-\lambda)}{6(1-\lambda)}\left(\left|a_{k}\right|+\left|b_{k}\right|\right) r
\end{gathered}
$$

and so

$$
\begin{gathered}
|f(z)| \geq 1+\left|b_{1}\right|-\frac{6(1-\lambda)}{2^{n}(3-\lambda)} \sum_{k=2}^{\infty} A\left[\frac{2 k-\lambda-1}{1-\lambda}\left|a_{k}\right|+\frac{2 k+\lambda+1}{1-\lambda}\left|b_{k}\right|\right] r \\
\leq 1+\left|b_{1}\right|-\frac{3(1-\lambda)}{2^{n}(3-\lambda)}\left[1-\frac{3+\lambda}{1-\lambda}\left|b_{1}\right|\right] r \\
=1+\left|b_{1}\right|-\frac{6}{2^{n}}\left[\frac{1-\lambda}{3-\lambda}-\frac{3+\lambda}{3-\lambda}\right] r
\end{gathered}
$$




$$
=\left(1+\left|b_{1}\right|-\frac{6}{2^{n}}\left[\frac{1-\lambda}{3-\lambda}-\frac{3+\lambda}{3-\lambda}\right] r\right.
$$

where $A=\frac{k^{n}(-1)^{k-1}}{(2 k-1)(k-1) !}$

On the other hand which complete the proof.

Theorem 4:Let $f=h+\bar{g}$, where $h$ and $g$ are given by (1). Then $f \in \operatorname{clcoH} \bar{T}_{H, n}(\lambda)$ if and only if

$$
f(z)=\sum_{k=1}^{\infty}\left(X_{k} h_{k}+Y_{k} g_{k}\right)
$$

where

$$
\begin{gathered}
h_{1}(z)=z \\
h_{k}(z)=z+\frac{1-\lambda}{\frac{k^{n}(-1)^{k-1}}{(2 k-1)(k-1) !}[2 k-\lambda-1]} z^{k} \quad(k=2,3, . .), \\
g_{k}(z)=z+\frac{k^{n}(-1)^{k-1}}{(2 k-1)(k-1) !}[2 k+\lambda+1] \bar{z}^{k} \quad(k=1,2,3, . .),
\end{gathered}
$$

$\sum_{k=1}^{\infty}\left(X_{k}+Y_{k}\right), X_{k} \geq 0$ and $Y_{k} \geq 0$. In particular, the extrem points of the class $\bar{T}_{H, n}$ are $\left\{h_{k}\right\}$ and $\left\{g_{k}\right\}$ respectively.

Proof. For a function of the form (9), we have

$$
\begin{gathered}
f(z)=\sum_{k=1}^{\infty}\left(X_{k} h_{k}+Y_{k} g_{k}\right) \\
=\sum_{k=1}^{\infty}\left(X_{k}+Y_{k}\right)(z)+\sum_{k=2}^{\infty} \frac{1-\lambda}{\frac{k^{n}(-1)^{k-1}}{(2 k-1)(k-1) !}[2 k-\lambda-1]} X_{k} z^{k}+\sum_{k=1}^{\infty} \frac{1-\lambda}{\frac{k^{n}(-1)^{k-1}}{(2 k-1)(k-1) !}[2 k+\lambda+1]} Y_{k} \bar{z}^{k} \\
=z+\sum_{k=2}^{\infty} \frac{1-\lambda}{\frac{k^{n}(-1)^{k-1}}{(2 k-1)(k-1) !}[2 k-\lambda-1]} X_{k} z^{k}+\sum_{k=1}^{\infty} \frac{1-\lambda}{\frac{k^{n}(-1)^{k-1}}{(2 k-1)(k-1) !}[2 k+\lambda+1]} Y_{k} \bar{z}^{k}
\end{gathered}
$$

But

Thus $f \in \operatorname{clco} H \bar{T}_{H, n}(\lambda)$.

$$
\begin{gathered}
\sum_{k=2}^{\infty} \frac{\frac{k^{n}(-1)^{k-1}}{(2 k-1)(k-1) !}[2 k-\lambda-1]}{1-\lambda}\left[\frac{1-\lambda}{\frac{k^{n}(-1)^{k-1}}{(2 k-1)(k-1) !}[2 k-\lambda-1]} X_{k}\right]+ \\
\sum_{k=2}^{\infty} \frac{\frac{k^{n}(-1)^{k-1}}{(2 k-1)(k-1) !}[2 k+\lambda+1]}{1-\lambda}\left[\frac{1-\lambda}{\frac{k^{n}(-1)^{k-1}}{(2 k-1)(k-1) !}[2 k+\lambda+1]} X_{k}\right] \\
\sum_{k=2}^{\infty} X_{k}+\sum_{k=1}^{\infty} Y_{k}=1-X_{1} \leq 1
\end{gathered}
$$

Conversely, suppose that $f \in \operatorname{clcoH} \bar{T}_{H, n}(\lambda)$. Set

$$
X_{k}=\frac{\frac{k^{n}(-1)^{k-1}}{(2 k-1)(k-1) !}[2 k-\lambda-1]}{1-\lambda}\left|a_{k}\right| \quad(k=2,3, . .),
$$

and

$$
Y_{k}=\frac{\frac{k^{n}(-1)^{k-1}}{(2 k-1)(k-1) !}[2 k+\lambda+1]}{1-\lambda}\left|b_{k}\right| \quad(k=1,2,3, . .),
$$

Then by the inequality Theorem 1 ,we have $0 \leq X_{k} \leq 1(k=2,3, .$.$) and 0 \leq Y_{k} \leq 1(k=1,2,3, .$.$) . Define$ $X_{1}=1-\sum_{k=2}^{\infty} X_{k}-\sum_{k=1}^{\infty} Y_{k}$ and note that $X_{1} \geq 0$. Thus we obain $f(z)=\sum_{k=2}^{\infty} X_{k} h_{k}+Y_{k} g_{k}$. This completes the proof of Theorem 4 . 


\section{Convolution and Convex Combinations}

For two harmonic functions

$$
\begin{gathered}
f(z)=z+\sum_{k=2}^{\infty} a_{k} z^{k}+\sum_{k=1}^{\infty} \bar{b}_{k} \bar{z}^{k} \\
F(z)=z+\sum_{k=2}^{\infty} A_{k} z^{k}+\sum_{k=1}^{\infty} \bar{B}_{k} \bar{z}^{k}
\end{gathered}
$$

we define their convolution

$$
(f * F)(z)=z+\sum_{k=2}^{\infty} a_{k} A_{k} z^{k}+\sum_{k=1}^{\infty} \overline{b_{k} B_{k}} \bar{z}^{k},
$$

using this definition, we show that the class $\bar{T}_{H, n}(\lambda)$ is close under convolution.

Theorem 5. Let $f, F \in \bar{T}_{H, n}(\lambda)$.Then $f * F \in \bar{T}_{H, n}(\lambda)$.

Proof: We note that $\left|A_{k}\right| \leq 1$ and $\left|B_{k}\right| \leq 1$.Now for the convolution $f * F$ we have

$$
\begin{aligned}
& \sum_{k=2}^{\infty} \frac{\frac{k^{n}(-1)^{k-1}}{(2 k-1)(k-1) !}[2 k-\lambda-1]}{1-\lambda}\left|A_{k} a_{k}\right|+\sum_{k=1}^{\infty} \frac{\frac{k^{n}(-1)^{k-1}}{(2 k-1)(k-1) !}[2 k+\lambda+1]}{1-\lambda}\left|B_{k} b_{k}\right| \\
& \leq \sum_{k=2}^{\infty} \frac{\frac{k^{n}(-1)^{k-1}}{(2 k-1)(k-1) !}[2 k-\lambda-1]}{1-\lambda}\left|a_{k}\right|+\sum_{k=1}^{\infty} \frac{\frac{k^{n}(-1)^{k-1}}{(2 k-1)(k-1) !}[2 k+\lambda+1]}{1-\lambda}\left|b_{k}\right| \leq 1
\end{aligned}
$$

Therefore $f * F \in \bar{T}_{H, n}(\lambda)$, which complete the proof.

We show that the class $\bar{T}_{H, n}(\lambda)$ is closed under convex combination of its members.

Theorem 6:The class $\bar{T}_{H, n}(\lambda)$ is closed under convex combination.

Proof:For $(i=1,2,3, \ldots)$ let $f_{i} \in \bar{T}_{H, n}(\lambda)$ where $f_{i}(z)$ is given by

$$
f(z)=z+\sum_{k=2}^{\infty} a_{k} z^{k}+\sum_{k=1}^{\infty} \bar{b}_{k} \bar{z}^{k}
$$

Then by Theorem 1 , we have

$$
\sum_{k=2}^{\infty} \frac{\frac{k^{n}(-1)^{k-1}}{(2 k-1)(k-1) !}[2 k-\lambda-1]}{1-\lambda}\left|a_{k_{i}}\right|+\sum_{k=1}^{\infty} \frac{\frac{k^{n}(-1)^{k-1}}{(2 k-1)(k-1) !}[2 k+\lambda+1]}{1-\lambda}\left|b_{k_{i}}\right| \leq 1
$$

For $\sum_{i=1}^{\infty} t_{i}=1,0 \leq t_{i} \leq 1$, the convex combination of $f_{i}$ may be written as

$$
\sum_{i=1}^{\infty} t_{i} f_{i}=z+\sum_{k=2}^{\infty}\left(\sum_{i=1}^{\infty} t_{i} a_{k_{i}}\right) z^{k}+\sum_{k=2}^{\infty}\left(\sum_{i=1}^{\infty} t_{i} \overline{b_{k_{i}}}\right) z^{k}
$$

Then by Theorem 1 , we have

$$
\begin{aligned}
& \sum_{k=2}^{\infty} \frac{\frac{k^{n}(-1)^{k-1}}{(2 k-1)(k-1) !}[2 k-\lambda-1]}{1-\lambda}\left|\sum_{i=1}^{\infty} t_{i} a_{k_{i}}\right|+\sum_{k=1}^{\infty} \frac{\frac{k^{n}(-1)^{k-1}}{(2 k-1)(k-1) !}[2 k+\lambda+1]}{1-\lambda}\left|\overline{\sum_{i=1}^{\infty} t_{i} b_{k_{i}}}\right| \\
& \leq \sum_{t=1}^{\infty} t_{1}\left(\sum_{k=2}^{\infty} \frac{\frac{k^{n}(-1)^{k-1}}{(2 k-1)(k-1) !}[2 k-\lambda-1]}{1-\lambda}\left|a_{k_{i}}\right|+\sum_{k=1}^{\infty} \frac{\frac{k^{n}(-1)^{k-1}}{(2 k-1)(k-1) !}[2 k+\lambda+1]}{1-\lambda}\left|b_{k_{i}}\right|\right) \\
& \leq \sum_{t=1}^{\infty} t_{1}=1
\end{aligned}
$$

Therefore $\sum_{i=1}^{\infty} t_{i} f_{i} \in \bar{T}_{H, n}(\lambda)$ 


\section{Conclusions}

Varying various parameter involved in class $f \in \bar{T} S_{H, n}(\lambda)$,known or new result could be arrived at.

\section{REFERENCES}

[1] M. Abramowitz and I. A. Stegun, (Eds), Handbook of Mathematical functions with Formulas, raphs, and Mathematical Tables, Dover Publications Inc., New York, 1965.

[2] O.P.Ahuja, R.Aghalary and S.B.Joshi, Harmonic univalent functions associated with k-uniformly starlike functions, Math. Sci. Res. J., 9(1) (2005), 9-17.

[3] H. Alzer, Error function inequalities, Adv. Comput. Math., 33 (2010), 349-379.

[4] J.Clunie and T.Sheil-Small, Harmonic univalent functions, Ann. Acad. Sci. Fen. Ser. A1 Math., 36 (1991), 13-16.

[5] D. Coman, The radius of starlikeness for error function, Stu. Uni. Babes-Bolyai Math., 36 (1991), 13-16.

[6] R.M.El-Ashwah and M.K.Aouf, A certain convolution approach for subclasses of univalent harmonic functions, Bull. Iran. Math. Soc., 41 (2015), 739-748.

[7] A. Elbert and A. Laforgia, The zeros of the complementary error function, Numer. Algorithms, 49 (2008), 153-157.

[8] A.M.Gbolagade, I.T.Awolere and A.O.Fadare, Goodman-Ronning type class of harmonic error function using Salagean operator, Electron. J. Math. Anal. Appl. 6(2) (2018), 307-316.

[9] J.M.Jahangiri, Harmonic functions starlike in the unit disk, J. Math. Anal. Appl., 235 (1999), 470-477.

[10] A.T.Oladipo, Coefficient inequality for subclass of analytic univalent functions related to simple logistic activation functions, Math. Std. Uni. Babes-Bolyai 61 (2016), 45-52.

[11] C. Ramachandran and K. Dhanalakshmi and L. Vanitha, Hankel determinant for a subclass of analytic functions associated with error functions bounded by conical regions, Int. J. Math. Anal. 11 (2017), 571-581.

[12] Salagean Gr..St (1981): Subclsses of univalent functions, Complex Analysis, Fifth Romanian - Finish Seminar, Lect. Notes Math. 1013, Springer- Verlag 1983, 362-372.

[13] P. Sharma, Goodman-Ronning type operators, class of harmonic univalent functions invoving convolutional, Int. J. Math. Archive, 3 (3) (2012), 1211-1221. 